\title{
Advanced Channeling Technologies in Plasma and Laser Fields
}

\author{
Sultan B. Dabagov ${ }^{\star}$ \\ ${ }^{1}$ INFN Laboratori Nazionali di Frascati, Frascati (RM), Italy \\ ${ }^{2}$ NR Nuclear University MEPhl, Moscow, Russia \\ ${ }^{3}$ PN Lebedev Physical Institute, Moscow, Russia
}

\begin{abstract}
Channeling is the phenomenon well known in the world mostly related to the motion of the beams of charged particles in aligned crystals. However, recent studies have shown the feasibility of channeling phenomenology application for description of other various mechanisms of interaction of charged as well as neutral particle beams in solids, plasmas and electromagnetic fields covering the research fields from crystal based undulators, collimators and accelerators to capillary based X-ray and neutron optical elements. This brief review is devoted to the status of channeling-based researches at different centers within international and national collaborations. Present and future possible developments in channeling tools applied to electron interactions in strong plasma and laser fields will be analyzed.
\end{abstract}

\section{Introduction}

As known the beam channeling in crystals takes place during relativistic charged particles motion in periodic structures close to the main crystallographic directions, which define the crystal axes or planes. For a relativistic particle traveling almost parallel to that directions the potential of interaction between the electron and a set of the lattice atoms (ions) could be averaged along the propagation direction. The interaction potential formed at these conditions might limit transverse motion of the projectile within well defined channels, i.e. relativistic particle becomes undulating in transverse plane at relativistic longitudinal motion down to the long narrow channel $[1,2]$.

For more than 50 years of intense studies the basics of crystal channeling for charged beams have been in details defined and described in many scientific papers and books, discussed in a number of conferences and workshops (see, for instance in [3-14] ). Presently crystal channeling is known as useful technique for beam steering $[15,16]$, while the related to crystal channeling phenomena are promising candidates for coherent radiation sources [17]. Moreover, the phenomenology of beams channeling becomes very useful to describe neutral beams handling via various beam guiding structures $[18,19]$. Besides, channeling conditions could be realized for particles not only in medium (crystals [20], capillaries [21, 22] and nanotubes [23-25], plasma [26-29]) but also in high intensity electromagnetic fields of specific configurations [30].

\footnotetext{
^e-mail: sultan.dabagov@lnf.infn.it
}

\section{Charged and Neutral Beams Channeling}

\subsection{Crystal channeling}

Channeling phenomenon was discovered in the beginning of 1960th at studying the passage of ion beams through crystals. As a result of computer simulations of charged particles penetration into crystals, Robinson, Oen and Beeler, Besco [31, 32] explained the effect of anomalous large penetration for fast ions moving with initial velocities close to principal axial directions. Almost simultaneously the newly predicted phenomenon ${ }^{1}$ was revealed experimentally [33, 34]. In 1965 Lindhard explained the existence of new phenomenon [1]; that work, presently well known as a classical one, has stimulated the great interest of physicists to the effect of channeling.

The channeling effect is observed when fast particles penetrate into a crystal under small angles (less than socalled Lindhard angle, the critical angle of channeling) to crystallographic planes (planar channeling) or axes (axial channeling). Channeled projectile penetrates along planes or axes into a crystal without significant energy loss at the distance, which is much larger than the corresponding distance in the case of amorphous solids. To describe this effect the model of continuous interaction potential, when fast particle interacts with the averaged field of atoms placed on a plane or axis instead of the field of separate atoms of a crystal was suggested. The corresponding averaged fields were called "continuous potentials" of the planes or axes that form well defined planar or axial channels, respectively, while the motion in that potentials - "channeling" motion.

\footnotetext{
${ }^{1}$ In reality for many years before this phenomenon was known as unusual deep penetration of ions at defined crystal orientations taking place at ion doping processes
} 
The classical channeling theory for charged heavy particles as well as the quantum theory was developed in successive works. Later on Andersen with coauthors have shown that channeling effect takes also place for electrons and positrons traversing crystals [35]. Further development of channeling theory has been mainly provoked by Kumakhov's work [17], in which powerful spontaneous $\gamma$ radiation by relativistic channeled particles has been predicted. It appears as a result of a classical charged particle undulation in the averaged channeling potential. Later on the classical approach was extended to quantum one, which explains the radiation process via transitions between quantum states in the potentials of atomic rows and planes (for Refs. see in the reviews [36-38]).

\subsection{Beam steering for collimation and extraction}

One of the rapidly developing application of channeling phenomenon relates to the problem of the heavy particles beam shaping (deflection, steering, splitting) in the modern accelerator facilities. The use of optimally bent crystals to fulfill the channeling condition has been first suggested in $[15,16]$ to deflect the charge particle beams over the angle much exceeding the critical angle of channeling. Indeed, when a crystal is bent along crystallographic planes, the channeled particle moves along bent channels and it can be deflected from initial direction at large angles (much exceeding the critical channeling angle).

At present the use of planar channeling in bent crystals to control a charge particle beam is of great interest as one of the accelerator and storage ring techniques for beam collimation. For the last decade several technologies for crystal collimation have been proposed and developed (single bent crystals and strip crystals in various configurations). During technological test measurements new phenomena for beam deflection have been observed (channeling, volume capture, volume reflection, etc) [39-44]. While for a long period the experiments have been dealing with the proton beams of $400 \mathrm{GeV} / \mathrm{c}$ at SPS CERN revealing the fine features of proton channeling, like beam mirroring, focusing, collimation, the last measurements were performed for LHC proton beams of $6.5 \mathrm{TeV} / \mathrm{c}$ that prove relativistic proton channeling [45]. Channeling-based parasitic beam extraction for high energy particles at present accelerator facilities has been recently proposed within the EU Crysbeam project [46] that aims in developing an alternative technique, which will be able to substitute massive magnetic kickers.

Recent studies have also shown rather efficient beam deflection for light particles. Indeed, the tests performed at MAMI and SLAC as well allowed observing very efficient sub-GeV (855 MeV MAMI) and multi-GeV (3.5 and $6.3 \mathrm{GeV}$ ) electron beam steering [47-49]. Simultaneously with the technique application, theoretical background for the beam propagation in bent crystal planar channels has been developed. Within collaboration with Tomsk Polytechnic University a new computer code to simulate the processes of relativistic proton/ion beams propagation into various crystals have been proposed and developed in order to characterize the beam deflection inside bent crys- tals [50-53]. This activity takes place within international CERN project UA9 and aims in LHC beams collimation based on crystal channeling phenomenon. UA9 LNF team has proposed to use parametric X-ray radiation (PXR) for diagnostics of both beam-crystal orientation and crystal quality; first PXR measurements for SPS CERN protons in a bent crystal have been performed and successfully proved the predictions for PXR making evident the peak structure in the radiation both spectral and angular distributions [54-56]. A new phenomenon on effective deflection of relativistic hadron and lepton beams by crystal miscut surface has been predicted $[57,58]$. Due to extremely fine structure of the miscut surface the phenomenon might be applied for future accelerators dealing with nanoscale emittance.

Another very important application of charged beam channeling relates, based on undulatory motion at channeling, to the possibility of creating powerful radiation sources in X-ray and $\gamma$-ray frequencies. This topic has been strongly investigated since the middle of 1970s, and till now remains of great interest for the researchers resulting in a number of new brilliant ideas. It would be difficult to review this subject in a short paper, hence, I do not discuss it in this work.

\subsection{X-ray and neutron channeling in micro- and nanoguides}

Some types of processes based on the self-organization of materials that have recently attracted considerable interest because of the possibility of preparing fine patterns of nanometer dimensions over larger areas, can be used for the fabrication of X-ray waveguides [59, 60]. Among them the formation of highly-ordered aligned carbon nanotubes and ordered arrays of uniform-sized porous in alumina is of great interest. X-ray propagation in nanochannels [59] is important due to potential applications in $\mathrm{X}$ ray optics. A special feature of these structures is a long, hollow, inner cavity, which could act as a channel for selective radiation penetration, similar to channeling of charged particles in crystals (see [18] and Refs. therein); nanochannels can be considered as capillaries (the base of capillary/polycapillary optical elements [61-63]). Research in X-ray propagation in capillary structures shows that diminishing the capillary internal radius from microns to nanometers results in a change of the character of radiation propagation, from the surface channeling in microcapillaries down to bulk channeling in nanocapillaries. Numerical simulations [64, 65] have shown that carbon nanotubes will act as soft X-ray waveguides and support modes of propagation when coated with various materials [66]. Other researchers have already demonstrated experimentally the coherent propagation of X-rays in a planar waveguide with a tunable air gap [67]. The angular dependence of the intensity of characteristic $\mathrm{K}_{\alpha}$ radiation in carbon vs the aligned carbon nanotubes orientation suggests the possibility of X-ray channeling as well as radiation diffraction on nanotube multiwalls [68].

The modes of radiation propagation in a waveguide are revealed at interference between the incident and reflected 
waves forming a standing wave pattern [59]. However, it becomes constructive just for specific angles. This phenomenon, valid for reflection from a flat surface, takes place just in the vicinity of the surface. Similar phenomena could be observed at radiation reflection from a curved surface (so called 'whispering modes') [69]. Strong radiation redistribution also takes place behind capillary systems (which is actually a simple example of the curved surface system); some structural features in the distribution are due to the geometry of the system (typically, hexagon type in the transverse cross section). However, some fine features could not be interpreted by ray optics, and require solution of the wave equation of radiation propagation.

The interference phenomena were observed for X-rays propagating in capillary structures practically 25 years ago. A first theoretical note regarding such a possibility for capillary optical systems, with X-ray interference behind capillaries, was published as an internal note [70]. After that, during 1993-94 at the S-60 LPI synchrotron ${ }^{2}$ the phenomenon was observed and successfully studied in a set of experiments on propagation of synchrotron radiation through transversely-ordered polycapillary optical elements (channels sizes of $\sim 100 \mu \mathrm{m}$, radiation wavelength $\sim 1 \div 10 \AA$ ). Then, the first paper appeared [71] with the wave interpretation of the features recorded. Phenomenology of the phenomenon given in [72] has shown that the fine features of $\mathrm{X}$-ray propagation in micron-size channels, which have been observed behind capillary structures, can be explained in view of the radiation interference due to the various channels curvatures. Later the trapped radiation propagation in the very vicinity of a surface was carefully studied in a number of papers [73-81] where the wave theory of $\mathrm{X}$ radiation propagation along a curved surface was developed (for more complete list of citations, see Refs. in [18]).

\section{Channeling in a Plasma-Ion Cavity}

Recent studies have shown that interaction of intense ultrashort laser pulses in a medium can be used as a source of compact electron beams with successful beams acceleration over short distances. Studying the propagation of nonlinear plasma electromagnetic waves being of great practical interest due to the progress in laser technologies $[84,85]$ has shown that powerful ultrashort laser pulses propagating in a plasma can accelerate the plasma electrons to ultra-relativistic energies (so called "laser wakefield acceleration")[86-89]. Considering the problem of powerful ultrashort laser pulse interaction with a plasma based on numerical simulations, a model for a strongly nonlinear regime of interaction of intense laser pulses with plasmas has been proposed. The model has demonstrated that in the process of laser interaction with the plasma the

\footnotetext{
${ }^{2}$ The cyclic electron accelerator S-60 LPI is used as a source of synchrotron radiation (SR) in vacuum ultraviolet (VUV) and soft X-ray spectral regions. The main characteristics are the maximum electron energy of $630 \mathrm{MeV}$, the electron orbit radius of $2 \mathrm{~m}$, the average current in the orbit of $130 \mathrm{~mA}$, the maximum of the SR spectrum is $0.66 \mathrm{keV}$. The synchrotron has been designed in the beginning of 1950s by Vladimir Veksler.
}

electrons, due to the ponderomotive forces, are displaced from the region at the higher laser intensity. This process has been described within the phenomenology of "moving bubble" [90] (Fig. 1). Indeed, the laser pulse propagating in the direction $\mathrm{Oz}$ pushes out quasi-free plasma electrons that results in creating a nearly spherical moving cavity, "bubble". This bubble, as said, is free of electrons and, hence, represents a rather big positive charge moving with the velocity of a laser pulse. Thus, this bubble forms laser-pulse-oriented field gradient, or, in other words, an plasma-ion channel, which is a distinct potential well for electrons. This potential can trap the plasma electrons consistently accelerating them.

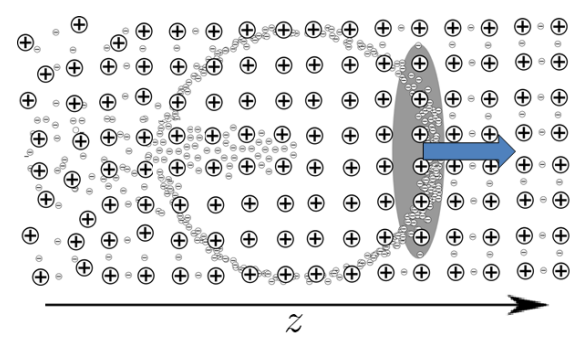

Figure 1. Schematic drawing of a strongly nonlinear regime of interaction of an ultrashort laser pulse with a plasma. Laser pulse propagating down the $\mathrm{Oz}$ axis with the velocity close to the light speed in vacuum produces an ion cavity, which involves plasma electrons into corresponding plasma-ion channel

The equations of electron motion in above described field at the condition that longitudinal momentum much exceeds transverse one, $p_{z}>>\left|\mathbf{p}_{\perp}\right|$, can be written in the following way

$$
\frac{d p_{z}}{d t} \approx-2 \pi e^{2} n_{0}\left(z-v_{l} t\right), \frac{d \mathbf{p}_{\perp}}{d t} \approx-2 \pi e^{2} n_{0} \mathbf{r}_{\perp},
$$

where $e$ and $n_{0}$ are the electron charge and the charge concentration in a medium, correspondingly, $v_{l}$ is the propagation velocity of a laser pulse. The force responsible for the electron acceleration is proportional to $\sim\left(z-v_{l} t\right)$. Hence, for electrons moving at a speed greater than the ion bubble speed the force exerted by the latter slows down the electrons. On the contrary, electrons moving at a speed less than the cavity speed become accelerated by the same force (see in $[82,83]$ and Refs. therein). Neglecting electrons acceleration down the channel at a slight difference between the velocities of electrons and ion chamber, the moving bubble can be presented by an infinite cylindrical plasma-ion cavity characterized by the following field potentials (for an infinite cylindrical channel containing "frozen" ions)

$$
\left\{\begin{array}{l}
\mathbf{A} \approx 0, \\
\varphi=-\pi n_{0} e r_{\perp}^{2},
\end{array}\right.
$$

Obviously, we deal with a long ion cavity, plasma-ion channel, characterized by quasi axially-symmetric potential well that binds plasma electrons. The bound projectile can be considered as a channeled one. Assuming relativistic electron motion down to the ion channel with transverse 
to the $O z$ axis oscillations, we can write the Hamiltonian in the form

$$
H=E_{z}+\frac{c^{2} p_{\perp}^{2}}{2 E_{z}}-e \varphi,
$$

where $E_{z}=c \sqrt{p_{z}^{2}+m^{2} c^{2}}$ is the longitudinal energy and the potential $\varphi$ is given by Eq.(2). As it results from the equation of motion, a channeled electron moving fast down to the channel becomes undulating in the transverse plane with the frequency of oscillations $\omega_{0}^{2}=2 \pi e^{2} n_{0} c^{2} / E_{z}$ (for typical electron longitudinal energies $\sim 1 \mathrm{GeV}$ and plasma concentrations $\sim 10^{19} \mathrm{~cm}^{-3}$ this frequency is of the order of $\omega_{0} \approx 4 \cdot 10^{12} \mathrm{~s}^{-1}$ ), characteristic trajectories of such motion are shown in Fig. 2.
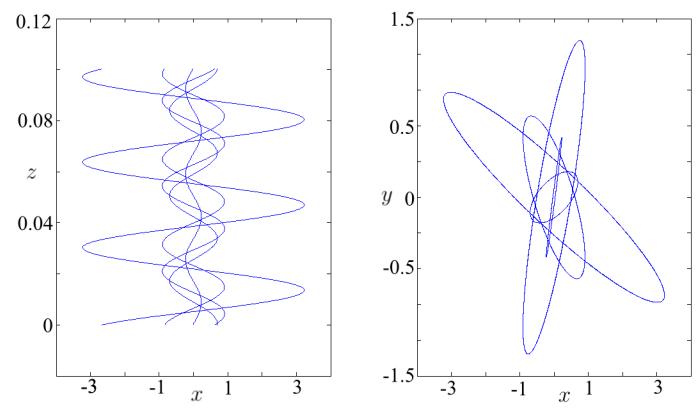

Figure 2. The electron trajectory in two transverse planes at different initial conditions. The graphs show that initial conditions determine the projectile trajectory. The longitudinal coordinate $z$ is given in $\mathrm{cm}$, while the vertical coordinates $x, y$-in $10^{-5} \mathrm{~cm}$.

Due to transverse undulations, the electron emits electromagnetic radiation mostly of X-ray frequencies, and we can define this type of radiation as channeling radiation of electrons in a plasma-ion cavity (radiation by electrons channeled in a plasma-ion channel) that simplifies essentially calculation of radiation functions. Utilizing channeling phenomenology for describing the radiation features the components of both vector $\mathbf{A}_{n}$ and scalar $\varphi_{n}$ potential expansions can be expressed in known sum of the spherical monochromatic waves as functions of the radiation frequency $\omega=\omega_{0} /\left(1-\beta_{z} n_{z}\right)$, where $\xi=n \omega\left(\mathbf{n}_{\perp}, \mathbf{v}_{0}\right) / \omega_{0} c$, and $\mathbf{n}$ is the unit vector in the direction of radiation. The angular distribution of electromagnetic radiation intensity is also defined by the potential parameters

$$
\frac{d I_{n}}{d \Omega}=\frac{\omega^{2} n^{2}}{2 \pi c}\left(\left|\mathbf{A}_{n}\right|^{2}-\left|\varphi_{n}\right|^{2}\right)
$$

The electron becomes trapped into the ion cavity under the following condition $\gamma_{z} m c>>p_{\perp}>>m c$ that results in main contribution to the radiation yield by high harmonics components and in a quasi-continuous spectrum of radiation as well. Simplified spectral intensity distribution in forward direction (relativistic projectiles emit in a narrow ahead directed cone) with respect to the longitudinal channel axis can be written as

$$
\left(\frac{d I}{d \omega}\right)_{\theta=0}=\frac{e^{2} \gamma_{z}^{4} \omega^{\prime 2}}{3 \pi^{3} c \omega_{0}}\left(\frac{3 \omega_{0}}{\omega_{m}}\right)^{2 / 3} f\left(\frac{\omega}{\omega_{m}}\right)
$$

where the function $f(\xi)$ is graphically presented in Fig. 3, and the maximum of radiation distribution function is at $\omega_{m}\left(\sim 10^{19} s^{-1}\right)$.

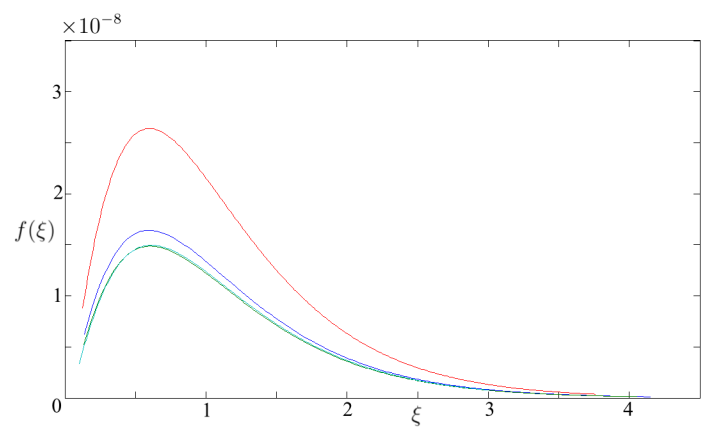

Figure 3. The function of spectral distribution of electromagnetic radiation by plasma-ion-channeled electron in forward direction at different initial parameters. The graph shows the shape of the spectrum to be almost independent of the initial conditions. Various curves correspond to various electron trajectories in the transverse plane $x O y: 1$ - a circular trajectory of the radius $r_{0}=10^{-4} \mathrm{~cm} ; 2$ - an elliptical trajectory with the semi-major axis of $a=r_{0} / 2$, which coincides with the $O x$ axis, and the semiminor axis of $b=r_{0} / 4$, which coincides with the $O y$ axis; 3 - an elliptical trajectory with the semi-major axis of $a=r_{0} / 3$, which coincides with the $O y$ axis, and the semi-minor axis of $b=r_{0} / 4$, which coincides with the $O x$ axis.

Despite the fact that the shape of the spectrum does not depend on the initial conditions, as seen from the graphs of Fig. 3 in the description of the radiation by channeled electrons the spatial distribution function of electrons depends strongly on initial conditions. This fact has a significant impact on coherent and incoherent radiation by the electron beam [91].

Described radiation is rather similar to channeling radiation in crystals [9]. However, on the contrary to channeling radiation in crystals when the electron is considered to be free along the channel direction (the continuous crystal potentials are transverse coordinate dependent), in our case, the electron is accelerated down the channel and known in literature as betatron radiation at laser wakefield acceleration $^{3}[27,92,93]$. The latter results in shifting the projectile's frequency for transverse oscillations during the electron channeling in a plasma-ion channel, and thus, the radiation spectrum becomes changed [94]. However, for $\mathrm{X}$-ray frequencies this change is not significant due to the fact that only electrons already being relativistic might be trapped in a channeling mode by the plasma-ion potential. Hence, for these frequencies the spectral radiation distribution by electrons in a plasma-ion channel, previously calculated and observed successfully, is close to that described in this work as channeling radiation.

\footnotetext{
${ }^{3}$ Obviously, the definition "betatron radiation" is far from the origin of radiation at the electron wakefield acceleration and can be accepted only because of the similarity of the trajectories for betatron electrons and wakefield accelerated ones.
} 


\section{Crossed Laser Field to Channel Charged Particles}

Nowadays the topic of electrons dynamics in crossed laser beams is gaining growing attention. Usually the case of standing electromagnetic wave being the result of two counter-propagating laser beams is considered. Kapitza and Dirac were the first who referred to the electron dynamics in such an interference field in well known paper [95] evaluating for the first time the electron beam diffraction in periodical field of an optical lattice. Since that many papers have been published proposing a tool for monitoring the electron beam size [96], a new-type free electron laser with optical undulator [97-101], a novel channeling radiation source [102-105] as well as several works clarifying processes taking place at interaction of charged beams in various periodical optical systems [106108]. Practically all those papers have been dedicated to the particle dynamics in the field of two laser beams.

There are much in common between particles dynamics in crossed laser beams and processes found in FEL undulators, betatron oscillations in plasma channels and crystal channeling. We should give a short remark on why the channeling point of view could be the most appropriate for considering the phenomenon. First of all, interference of two crossed laser beams creates electromagnetic field peaks and nodes, i.e. optical lattice, which is similar to the crystal lattice. This forms semblance of crystal lattice in absence of actual medium that becomes important due to the possibility of beam handling without inelastic scattering of the beam by the medium subsystems. Furthermore, averaging interaction of a particle with both crystal and optical lattices one derives effective potential responsible for particle channeling in these systems. And this descriptive similarity is not the only reason for treating the considered process as channeling.

The typically used ponderomotive force to describe interaction of a charged particle in a laser field is defined by the laser frequency and the gradient of the laser electrical field amplitude [109, 110]

$$
\mathbf{F}_{p}=\frac{e^{2}}{4 \omega_{0}^{2} m} \vec{\nabla}|\mathbf{E}(x)|^{2},
$$

where $m$ is the mass of electron, $\omega_{0}$ is the laser frequency and $\mathbf{E}(x)$ is the electrical field amplitude distribution over $x$-coordinate. However, this force depends not only on laser intensity but also on its polarization as well as on the energy of particle [106, 107]. Situation becomes even more complex when we deal with the field formed by two interfering laser waves [108].

The electron motion in optical lattice formed by crossed linearly polarized laser beams, in plane electromagnetic waves approximation, can be described in terms of particles channeling. In the region of two laser beams overlapping the ponderomotive force characterized by averaged effective potential affects charged particles. The ponderomotive potential forms planar (one-dimensional) potential wells (Fig. 4). This structure is similar in some way to the crystal lattice, seen by a high-velocity projectile, that is a set of crystal nodes, forming crystallographic planes and axes. The standing waves can be represented as the effective potential wells, the channels, periodically situated in a transverse plane that can trap the electron at specific conditions.

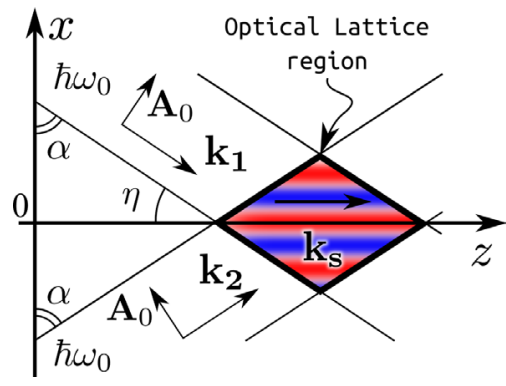

Figure 4. The general scheme of the considered system. Two crossed laser beams of the $\omega_{0}$ frequency with the wave vectors $\mathbf{k}_{\mathbf{1}}$ and $\mathbf{k}_{\mathbf{2}}$, which lie in the $x O z$ plane at the acute angles $\eta=\pi / 2-\alpha$ with respect to the $O z$ direction. The effective potential channels (shown in color) are parallel to $0 z$-axis.

To examine the bound motion of charged particles in optical lattice, Kapitza method [111] is rather effective at non-relativistic electron energies when classical approximation might be applied to the solution of the equation of electron motion. Within this approach, due to the presence of high frequency electromagnetic field, the electron trajectory at defined conditions looks like frequent oscillations $\delta x_{i}$ along a smooth curve $\bar{x}_{i}: x_{i}=\bar{x}_{i}+\delta x_{i}$. Neglecting the oscillations in the equation of motion (small by the def-

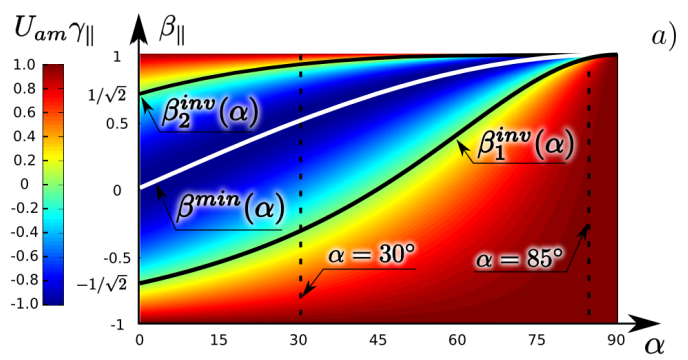

a)

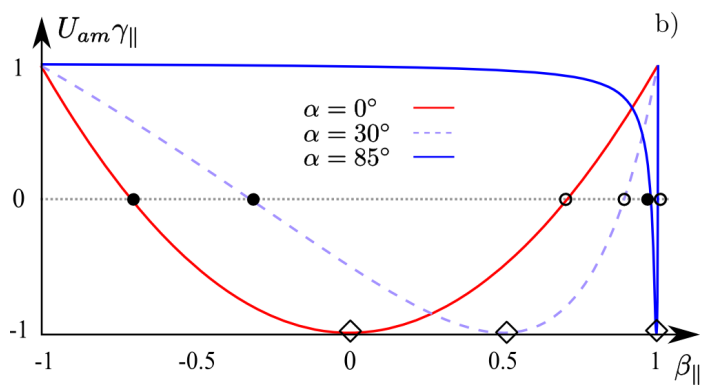

Figure 5. a) The averaged potential amplitudes normalized by $\left(e^{2} A_{0}^{2}\right) /\left(2 \gamma_{\|} m c^{2}\right)$ as a function of $\beta_{\|}$and $\alpha$ is shown in color. Inversion speed values $\beta_{1,2}^{i n v}$ as a functions of $\alpha$ are shown in black. The electron speed value for which at chosen $\alpha$ the value of $U_{a m} \gamma_{\|}$becomes minimal, $\beta^{\min }(\alpha)$, is shown in white. b) Normalized potential amplitudes for two p-polarized laser beams, crossed at different angles. The inversion speed values $\beta_{1}^{\text {inv }}$ are marked with $\bullet$, values of $\beta_{2}^{\text {inv }}-$ with $\circ$, and $\beta^{\text {min }}-$ with $\diamond$ for each $\alpha$. 
inition, $\delta x_{i} \sim e E_{0} / m \omega_{0}^{2}$ ), an effective potential energy of electron interaction in combined field can be defined. Applying some modification to Kapitza method the problem can be solved also for relativistic velocities [108] when after averaging by rapid oscillations one derives the effective potential expression

$$
\begin{aligned}
& U_{e f f}=-U_{a m} \cos (2 k x \cos \alpha), \\
& U_{a m}=\frac{e^{2} A_{0}^{2}}{2 \gamma_{\|} m c^{2}} \frac{\left(1+\cos ^{2} \alpha\right) \beta_{\|}^{2}-\cos (2 \alpha)-2 \beta_{\|} \sin \alpha}{\left(1-\beta_{\|} \sin \alpha\right)^{2}},
\end{aligned}
$$

where $\beta_{\|}=v_{0} / c$ and positive $\beta_{\|}$means that the electron moves in the direction of $\mathbf{k}_{s}=\mathbf{k}_{1}+\mathbf{k}_{2}$, while negative $\beta_{\|}$- in opposite direction; $A_{0}$ is the longitudinal vector potential amplitude. The above derived expression describes the effective potential in the region of two p-polarized laser beams crossed at arbitrary angle (Fig. 5). The motion equation could be linearized allowing its description via known elliptic integral trajectory characterized by both frequencies

$$
\Omega_{1}=2 k \cos \alpha \sqrt{U_{a m} / \gamma_{\|} m}, \Omega_{2}=\omega_{0}\left(1-\beta_{\|} \sin \alpha\right),
$$

where $\Omega_{1,2}$ are the frequencies for channeling oscillations (within standing wave structure) and for rapid oscillations due to the particle interaction with original laser field, respectively.

At $\alpha=0$ the beams are counter-propagating and the potential amplitude in this case becomes positive for $\left|\beta_{\|}\right|>$ $1 / \sqrt{2}$ and negative otherwise. This means that for an electron moving at the speed $\left|\beta_{\|}\right|=1 / \sqrt{2}=-\beta_{1}^{\text {inv }}=\beta_{2}^{\text {inv }}$ (so called "inversion speed") no periodic potential is formed. When the electron speed is $\left|\beta_{\|}\right|>1 / \sqrt{2}$, the regions of interference electric field peaks are scattering ones for it. And for $\left|\beta_{\|}\right|<1 / \sqrt{2}$ these regions are characterized by the attractive potentials. This potential inversion is observed only for p-polarized crossed laser beams.

The phase space trajectories projected onto the plane of transverse momentum and coordinate can be presented for both channeled and over-barrier electrons in the field of laser beams crossed as shown in Fig. 6, for instance, at the angle of $2 \eta=30^{\circ}\left(\alpha=75^{\circ}\right)$. The inner closed curves (in blue) correspond to channeled electrons with transverse energies less than potential well height. Overbarrier electrons (in red) are characterized by transverse energies greater than the barrier height and not limited within the channels. For electron with $\beta_{\|}=\beta^{\text {min }}$ the channel borders are situated at $x / d_{c h}=0.5+n$ and, correspondingly, the channel centers - at $x / d_{c h}=n$ (see Fig. 6a). On the contrary, electron with longitudinal velocity $\beta_{\|} \rightarrow 1>\beta_{2}^{\text {inv }}$ could be trapped by the channels, central axes of which are placed at $x / d_{c h}=0.5+n$ (see Fig. 6b), where $n=0, \pm 1, \pm 2, \ldots$.

Obviously, a particle oscillating in external field should radiate at frequencies related to the undulatory motion. The radiation spectrum of a charged particle emitting in a crossed laser field is characterized by two peak frequencies $\omega_{1,2}=\Omega_{1,2} /\left(1-\beta_{\|} \cos \theta\right)$ [105], where $\Omega_{1,2}$ were defined before by the expressions (8). Both of them being measured in the forward direction $(\theta=0)$ are shifted by
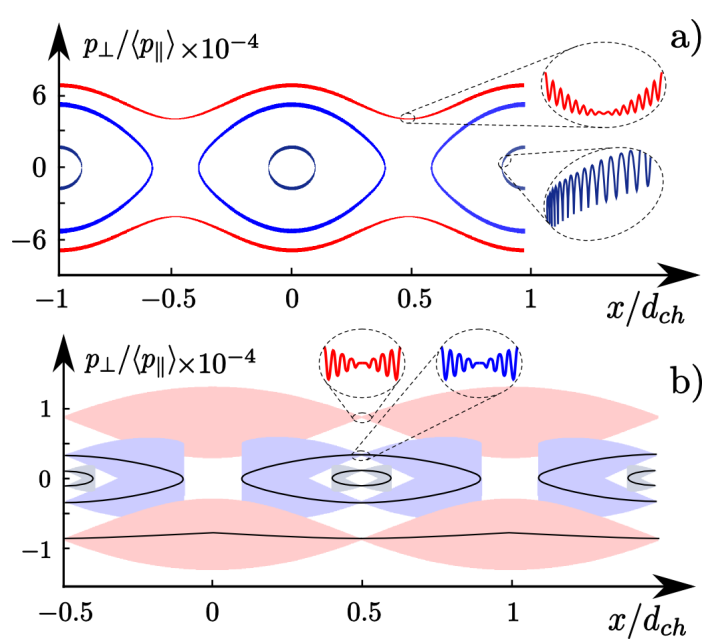

Figure 6. Simulated transverse phase-space trajectories for two extreme electron energies $-\beta_{\|}=\beta^{\min }$ (a) and $\beta_{\|}=0.99986$ (b). The channel has formed by two p-polarized lasers of $\lambda=800 \mathrm{~nm}$ wavelength and $5 \mathrm{TW} / \mathrm{cm}^{2}$ intensity for each one. The electron transverse momentum is normalized by the averaged longitudinal momentum, while the transverse coordinates - by the channel width $d_{c h} \equiv \lambda_{0} /(2 \sin \eta)$. Channeled (under-barrier) trajectories are shown in blue, while quasichanneled (over-barrier) - in red. The rapid oscillations could not be properly visualized and are outlined near the plots. The averaged phase space trajectories are also demonstrated in solid black lines.

the factor of $\sim 2 \gamma_{\|}^{2}$ due to the Doppler effect. The first one $\left(\omega_{1}\right)$ corresponds to slow channeling electron oscillations in the potential well of a system and does not depend on the laser frequency $\omega_{0}$. The $\omega_{2}$ radiation frequency is caused by the electron interaction with the original laser beams field.

Taking into account that relativistic particle emits radiation in a narrow forward directed solid cone $\left(\sim \gamma^{-1}\right)$, one could define the analytical expression for radiation spectrum of relativistic channeled electron moving near the bottom (center) of the cross-laser channel

$$
\frac{d P}{d \omega}=\sum_{i=1}^{2} \frac{e^{2} \Omega_{i}^{3} a_{i}^{2} \gamma_{\|}^{2}}{c^{3}} \zeta_{i}\left(1-2 \zeta_{i}+2 \zeta_{i}^{2}\right) \Theta\left(\pi N_{i}\left(1-\zeta_{i}\right)\right),
$$

where $\omega$ is the radiation frequency, $a_{1}$ is the amplitude of channeling oscillations, and $a_{2}$ is the amplitude of rapid oscillations, $N_{i}$ is the number of $\Omega_{i}$-frequency particle oscillations, $\zeta_{i}=\omega /\left(2 \gamma_{\|}^{2} \Omega_{i}\right)$, and $\Theta(x)=0.5+\mathrm{Si}(2 x) / \pi-$ $\sin ^{2}(x) / \pi x$. Total low-frequency power emitted by the channeled electron can be then evaluated by

$$
P[W]=\frac{2 e^{2} \Omega_{1}^{4} a_{1}^{2} \gamma_{\|}^{4}}{3 c^{3}} \approx 10^{-42}\left(I\left[\frac{W}{\mathrm{~cm}^{2}}\right]\right)^{2}
$$

for the channeling oscillations amplitude $a_{1} \approx 0.1 d_{c h}$. The channel width for the external laser wavelength of $\lambda_{0}=$ $1 \mu m$ crossed at $\eta=15^{\circ}$ would be $d_{c h} \equiv \lambda_{0} /(2 \sin (\eta)) \approx$ $2 \lambda_{0} \approx 2 \mu m$.

One should note that, first, both electrons and lasers parameters could be chosen so that the radiation wavelength is equal to the external laser one. Hence, this could 
be interesting as amplification mechanism for increasing external laser intensity. Moreover, due to rather wide tunable channel width it becomes possible to use a large bunch. In case of, for instance, $10^{10}$ electrons (monoenergetic, for simplicity), forming a bunch of several $\mu \mathrm{m}$ transverse width, low-frequency coherent radiation power is

$$
P[W] \approx 10^{-22}\left(I\left[\frac{W}{\mathrm{~cm}^{2}}\right]\right)^{2}
$$

As seen, in the case of initial laser intensity of $10^{17} \mathrm{~W} \mathrm{~cm}^{-2}$ the total low-frequency radiation power is estimated to be equal to $10^{12} \mathrm{~W}$.

The radiation spectrum classically calculated is characterized by two major frequencies: the first one is due to external laser field scattering on relativistic electron, while the second corresponds to the radiation of electron trapped by the effective potential well. The maximum radiation intensity falls on the frequency $\omega_{m}^{\text {rad }} \sim \gamma_{\|} \sqrt{I}$ defined by both electron energy and external laser intensity, while the integral radiated power depends on squared external laser intensity $P \sim I^{2}$. Combined with high radiation coherence for a channeled electron bunch it can result in a high intensity gain. The main issue here would be not only electron bunch low-frequency radiation coherency but also electrons cooling due to high-frequency radiation which would be considerable in strong fields.

The expressions for the effective potential and radiation yield were derived mostly for one-electron case. And the most interesting questions concerning beam dynamics, cooling and coherent radiation in crossed lasers are the subject of our current research that will help to determine the feasibility and conditions for creating tools based on the considered phenomena. However, in order to analyze the interaction of charged particles beam in laser optical lattices we have to use computer simulations that allows taking into account space charge interaction which involves all the beam particles. It should be underlined that in a beam we deal simultaneously with practically all possible solutions for a single particle motion that has been previously analyzed (Fig. 7). Moreover, only in multiple particle dynamics we can predict the peculiarities such as beam shaping, deflection, focusing.

\section{Conclusions}

For more than 50 years of its history the channeling phenomenon having started from trapped motion in aligned crystals has been observed at propagation of X-ray radiation and thermal neutrons in capillary structures, and presently becomes very useful to examine fine features of charged beam dynamics in laser and plasma based guides.

As shown the similarity of possible applications and effects may take place in optical lattice and in crystal channeling. The effective potential of both crystal and optical lattices could be very similar and both are capable of trapping electrons. So that channeled electron beams can be transported, focused and reflected by potential of both lattices.

Bending a crystal one obtains a tool for charged particles beams steering and bent laser channels might be also
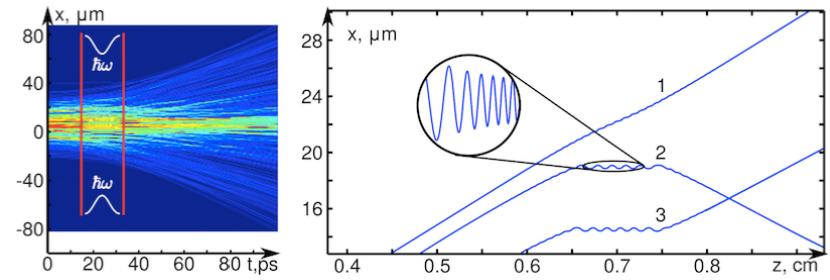

Figure 7. Numerical simulations for a Gaussian profile $\sim 1 \mathrm{pC}$ nondivergent electron beam of $\sim 2 \mathrm{MeV}$ interacting with a laser standing field of the Gaussian transverse profile with $\sigma_{z}=1 \mathrm{~mm}$. The laser fan has positioned at 23 ps from the initial electron beam position. The density color graph of electron beam passing through optical lattice depicts the particle time distribution. Curve 1 - non channeled particles, curve 2, 3 - channeled particles.

applied for this. Such channels can be formed by illuminating a curved reflecting surface with a laser at some angle that creates interference region with potential channels near the surface. Besides, recently researches have shown different regimes of charged particles dynamics in presence of intense laser fields that are analogous to crystal volume reflection, to both planar and axial crystal channeling and optical undulating.

Electrons deflection by curved laser channels, formed by laser grazing incidence on curved reflecting surface is a new research inspired by analogy of optical lattice channeling and crystal channeling, which could be done in bent crystals for charged particle beam steering. The reported derivation of curved laser channel effective potential is done for the region close to the reflecting surface and the resulting critical radius values for ultra-relativistic electrons are extremely high, but the idea may find application for moderate energies. In any case additional investigation is needed.

Channeling phenomenology applied to the description of wake-filed acceleration becomes very powerful tool that enables analyzing the dynamics of beam acceleration as well as the radiation by accelerated particles. Application of channeling technique can simplify prediction of various new effects.

Besides of beam shaping studies based on channeling technique, it is worthy to underline that particle channeling is always accompanied by rather strong radiation. Channeling motion provides high-frequency and low amplitude undulation of trapped particles that results in high flux electromagnetic emission of wide frequency range. The frequency is defined by the parameters of the beam and external field. In major cases this radiation, additionally to the interest of creating powerful tunable radiation source, might be of the reason for efficient beam cooling that is under our present investigations.

First of all, I would like to emphasize that I am extremely grateful to the organizers of the PPLA 2017 conference for the invitation to present the results of our recent studies on channeling of charged and neutral particles in strong external fields of various origin. 
I would like to acknowledge the work done by my young colleagues Alexey Dik and Evgeny Frolov. I have included in this short review the results of our common research for several last years.

This work was supported by the Competitiveness Program of National Research Nuclear University "MEPhI".

\section{References}

[1] J. Lindhard, Kgl. Dan. Vid. Selsk. Mat.-Fys. Medd., 34 (14) (1965) 1.

[2] D.S. Gemmell, Rev. Mod. Phys., 46 (1974) 129.

[3] M.A. Kumakhov, and G. Shirmer, Atomic Collisions in Crystals (G. \& B. Sci. Pub. Ltd, London) 1979.

[4] V.G. Baryshevsky, Channeling, Radiation and Reactions in Crystals at High Energies (BSU, Minsk) 1982 (in Russian).

[5] Y.-H. Ohtsuki, Charged Beam Interaction with Solids (Taylor \& Francis Ltd, London-New York) 1983.

[6] A.W. Saenz, and H. Uberall, Eds, Coherent Radiation Sources (Springer) 1985.

[7] V.A. Bazylev, and N.K. Zhevago, Radiation of Fast Particles in Matter and External Fields (Nauka, Moscow) 1987 (in Russian).

[8] N.P. Kalashnikov, Coherent Interactions of Charged Particles in Single Crystals. Scattering and Radiative Processes in Single Crystals. Harwood Acad. Pub. London and New York. 1988.

[9] M.A. Kumakhov, and F.F. Komarov, Radiation from Charged Particles in Solids (AIP, New York) 1989.

[10] A.I. Akhiezer, and N.F. Shuloga, High-Energy Electrodynamics in Matter (G. \& B. Sci. Pub., Amsterdam) 1996.

[11] V.N. Baier, V.M. Katkov, and V.M. Strakhovenko, High Energy Electromagnetic Processes in Oriented Single Crystals (World Scientific, Singapore) 1998.

[12] P. Rullhusen, X. Artru, and P. Dhez, Novel Radiation Sources Using Relativistic Electrons (World Scientific, Singapore) 1998.

[13] Proceedings of the "Channeling" conferences for the period of 2004-2016, Dabagov S.B., Ed.: Proc. of SPIE 5974 (2005); Proc. of SPIE 6634 (2007); Proc. of the 51st Workshop of the INFN Eloisatron Project, World Scientific, (2010); Nuovo Cimento C 34 (4) (2011); Nucl. Instr. Meth. B309 (2013); Nucl. Instr. Meth. B355 (2015); Nucl. Instr. Meth. B402 (2017).

[14] S.B. Dabagov, and M.N. Strikhanov, Eds., the books "Charged \& Neutral Particles Channeling Phenomena", MEPhI Pub., Moscow, ISBN 978-5-72621864-9 (2013); ISBN-978-5-7262-2232-5 (2016).

[15] E.N. Tsyganov, Fermilab TM-682, Batavia (1976).

[16] R. Carrigan, Phys. Rev. Lett.,35 (1975) 206.

[17] M.A. Kumakhov, Physics Letters A57 (1976) 17.

[18] S.B. Dabagov, Phys. Usp., 46 (2003) 1053.

[19] I. Bukreeva et al., Phys. Rev. Lett. 97 (2006) 184801.

[20] V.M. Biryukov, Y.A. Chesnokov, and V.I. Kotov, Crystal Channeling andIts Application at HighEnergy Accelerators (Springer, Berlin) 1997.
[21] M.A. Kumakhov and F.F. Komarov, Phys. Rep. 191 (5) (1990) 289.

[22] C. MacDonald, X-Ray Opt. Instr. 867049-17 (2010).

[23] N.K. Zhevago and V.I. Glebov, Phys. Lett. A310 (2003) 301.

[24] V.V. Klimov and V.S. Letokhov, Phys. Lett. A222 (1996) 424.

[25] A. Karabarbounis, S. Sarros, and C. Trikalinos, Nucl. Instr. Meth. B316 (2013) 160.

[26] E. Esarey et al., Phys. Rev. E 65, 056505 (2002).

[27] I. Kostyukov, S. Kiselev, and A. Pukhov, Phys. Plasmas 10 (2003) 4818.

[28] J. Faure et al., Phys. Plasmas 17, 083107 (2010).

[29] A.V. Dik, A.Z. Ligidov, and S.B. Dabagov, Nucl. Instr. Meth. B309 (2013) 210.

[30] E.N. Frolov, A.V. Dik, and S.B. Dabagov, Nucl. Instr. Meth. B309 (2013) 157.

[31] M.T. Robinson, and O.S. Oen, Phys. Rev. 132 (1963) 2385.

[32] J.R. Beeler, and D.S. Besco, J. Appl. Phys. 34 (1963) 2873.

[33] G.R. Piercy et al., Phys. Rev. Lett. 10 (1963) 399.

[34] H. Lutz, and R. Sizmann, Physics Letters 5 (1963) 113.

[35] J.U. Andersen, S.K. Andersen, and W.M. Augustyniak, Kgl. Dan. Vid. Selsk. Mat.-Fys. Medd., 39 (10) (1977) 1.

[36] V.V. Beloshitsky, and F.F. Komarov, Phys. Rep. 93 (1982) 117.

[37] R.A. Carrigan, Jr., and J.A. Ellison, Eds., Relativistic Channeling (Plenum Press, New York) 1987.

[38] S.B. Dabagov, and N.K. Zhevago, La Rivista del Nuovo Cimento 31 (9) (2008) 491.

[39] W. Scandale et al, Physics Letters B703 (2011) 547.

[40] W. Scandale et al, Physics Letters B726 (2013) 182.

[41] W. Scandale et al, Physics Letters B733 (2014) 366.

[42] W. Scandale et al, Physics Letters B734 (2014) 1.

[43] W. Scandale et al, Nucl. Instr. Meth. B338 (2014) 108.

[44] W. Scandale et al, Physics Letters B748 (2015) 451.

[45] W. Scandale et al, Physics Letters B758 (2016) 129.

[46] http://crysbeam.roma1.infn.it/index.html

[47] A. Mazzolari et al., Phys. Rev. Lett. 112 (2014) 135503

[48] U. Wienands et al., Phys. Rev. Lett. 114 (2015) 074801 .

[49] U. Wienands et al., Nucl. Instr. Meth. B402 (2017) 11. doi: 10.1016/ j.nimb.2017.03.097

[50] A. Babaev, G. Cavoto, and S.B. Dabagov, Nucl. Instr. Meth. B309 (2013) 120.

[51] A. Babaev, and S.B. Dabagov, Journ. of Physics: Conf. Ser. 357 (2012) 012032.

[52] A. Babaev and S.B. Dabagov, Eur. Phys. J. Plus 127 (2012) 62 .

[53] A. Babaev, and S. Dabagov, Il Nuovo Cimento C34 (4) (2011) 417.

[54] W. Scandale et al, Physics Letters B701 (2011) 180. 
[55] S.B. Dabagov, et. al., INFN-16-05/LNF, 17th March 2016.

[56] K.B. Korotchenko, Yu.L. Eykhorn, and S.B. Dabagov, Phys. Lett B774 (2017) 47-475.

[57] A. Babaev, G. Cavoto, and S.B. Dabagov, Nucl. Instr. Meth. B355 (2015) 356.

[58] A. Babaev, G. Cavoto, and S.B. Dabagov, JETP Letters 100 (9) (2014) 550.

[59] E. Spiller, and A. Segmuller, Appl. Phys. Lett. 24 (1974) 60.

[60] S.P. Pogossian, Opt. Comm. 114 (1995) 235.

[61] M.A. Kumakhov, and F.F. Komarov, Phys. Rep. 191 (1990) 289.

[62] P. Engstrøm, S. Larsson, and A. Rindby, Nucl. Instr. Meth. A302 (1991) 547.

[63] D.J. Thiel et al., Nucl. Instr. Meth. A317 (1992) 597.

[64] N.K. Zhevago, and V.I. Glebov, Phys. Lett. A250 (1998) 360.

[65] G.V. Dedkov, Nucl. Instr. Meth. B143 (1998) 584.

[66] P.A. Childs, and A.G. O’Neill, Physica E19 (2003) 153.

[67] M.J. Zwanenburg et al., Phys. Rev. Lett. 82 (1999) 1696.

[68] A.V. Okotrub et al., JETP Lett. 81 (2005) 34.

[69] A. Vinogradov et al., Sov. Phys. - Tech. Phys. 30 (1985) 335.

[70] S.B. Dabagov, Redistribution of X-rays Trapped in Bound States by Capillary Systems (Research Report of FIROS: Nalchik-Moscow, 1992).

[71] S.B. Dabagov et al., J. Synchrotron Rad. 2 (1995) 132.

[72] S.B. Dabagov, M.A. Kumakhov, and S.V. Nikitina, Phys. Lett. A203 (1995) 279.

[73] S.B. Dabagov, and M.A. Kumakhov, Proc. SPIE 2515 (1995) 124

[74] Yu.M. Alexandrov et al., Nucl. Instr. Meth. B134 (1998) 174.

[75] N. Artemiev et al., Phys. Scripta 57 (1998) 228.

[76] S.B. Dabagov et al., Appl. Opt. 39 (2000) 3338.

[77] S.B. Dabagov et al., Proc. SPIE. 3444 (1998) 486.

[78] C. Liu, and J.A. Golovchenko, Phys. Rev. Lett. 79 (1997) 788.

[79] S.V. Kukhlevsky et al., Nucl. Instr. Meth. B168 (2000) 276.

[80] G. Cappuccio, et al., Appl. Phys. Lett. 78 (2001) 2822.

[81] S.B. Dabagov, X-ray Spectrom. 32 (2003) 179.

[82] D. Giulietti, J. Phys.: Conf. Ser. 508 (2014) 012001.

[83] L. Torrisi, et al., J. Applied Phys. 107 (2010) 123303.

[84] G. Mourou, C. Barty, and M. Perry, Phys. Today 51 (1) (1998) 22.
[85] S.P.D. Mangles et al., Phys. Rev. Lett. 94 (2005) 24500.

[86] T. Tajima, and J.M. Dawson, Phys. Rev. Lett. 43 (1979) 267.

[87] S. Bulanov, V. Kirsanov, and A. Sakharov, Plasma Phys. 16 (1990) 543.

[88] A. Pukhov, S. Kiselyov, and I. Kostyukov, Appl. Phys. 6 (2006) 35 (in Russian).

[89] J. Faure et al., Nature 431 (2004) 541.

[90] E. Esarey, C. Schroeder, and P. Leemans, Rev. Mod. Phys. 81 (2009) 1229.

[91] A. Dik, and S.B. Dabagov, Izv VUZov 12 (2012) 77 (in Russian).

[92] A. Rousse, et al., Phys. Rev. Lett. 93 (13) (2004) 135005

[93] A. Curcio, and D. Giulietti, NIMB 355 (2015) 214.

[94] S. Corde, et al., Rev. Mod. Phys. 85 (2013) 045004.

[95] P.L. Kapitza, and P.A.M. Dirac, Math. Proc. Cambridge Philos. Soc. 29 (1933) 297.

[96] T. Shintake, Nucl. Instr. Meth. A311 (1992) 453.

[97] M.V. Fedorov, K.B. Oganesyan, and A.M. Prokhorov, Appl. Phys. Lett. 53 (1988) 353.

[98] P. Balcou, Eur. Phys. J. D 59 (2010) 525.

[99] I.A. Andriyash, P. Balcou, and V.T. Tikhonchuk, Eur. Phys. J. D 65 (2011) 533.

[100] I.A. Andriyash et al., Phys. Rev. Lett. 109 (2012) 244802

[101] I.A. Andriyash et al., J. Phys. Conf. Ser. 414 (2013) 012008 .

[102] M. Bertolotti, C. Sibilia, and L. Fuli, in Trends in Quantum Electronics, A.M. Prokhorov, I. Ursu, Eds. (Springer, Berlin Heidelberg, 1986).

[103] A. V. Andreev, and S. A. Akhmanov, JETP Lett. 53 (1991) 18.

[104] E.N. Frolov, A.V. Dik, and S.B. Dabagov, Nucl. Instr. Meth. B309 (2013) 157.

[105] E.N. Frolov, A.V. Dik, and S.B. Dabagov, J. Phys. Conf. Ser. 517 (2014) 012002.

[106] A.E. Kaplan, and A.L. Pokrovsky, Phys. Rev. Lett. 95 (2005) 053601.

[107] A.L. Pokrovsky, and A.E. Kaplan, Phys. Rev. A72 (2005) 043401

[108] S.B. Dabagov, A.V. Dik, and E.N. Frolov, Phys. Rev. ST 18 (2015) 064002.

[109] A.V. Gaponov, and M.A. Miller, Zh. Eksp. Teor. Fiz. 34 (1958) 242 (in Russian).

[110] B.M. Bolotovskii, and A.V. Serov, Usp. Fiz. Nauk. 37 (1994) 515.

[111] L.D. Landau and E.M. Lifshitz, Mechanics, Course of Theoretical Physics, Vol. 1, 3rd ed. (Elesevier, Amsterdam etc.) 1976. 